\title{
Hyers-Ulam Stability of Iterative Equation in the Class of Lipschitz Functions
}

\author{
Chao Xia and Wei Song \\ Department of Mathematics, Harbin Institute of Technology, Harbin, Heilongjiang 150001, China \\ Correspondence should be addressed to Chao Xia; xiachaomath@gmail.com
}

Received 14 March 2014; Revised 10 May 2014; Accepted 10 May 2014; Published 1 June 2014

Academic Editor: Krzysztof Ciepliński

Copyright (c) 2014 C. Xia and W. Song. This is an open access article distributed under the Creative Commons Attribution License, which permits unrestricted use, distribution, and reproduction in any medium, provided the original work is properly cited.

\begin{abstract}
Hyers-Ulam stability is a basic sense of stability for functional equations. In the present paper we discuss the Hyers-Ulam stability of a kind of iterative equations in the class of Lipschitz functions. By the construction of a uniformly convergent sequence of functions we prove that, for every approximate solution of such an equation, there exists an exact solution near it.
\end{abstract}

\section{Introduction}

It is pointed out that the theory of Ulam's type stability (also quite often connected, e.g., with the names of Bourgin, Găvruţa, Aoki, Hyers, and Rassias) is a very popular subject of investigations at the moment in [1]. For more details of Ulam's type stability, we refer the audience to [1-3]. In [1], the authors present a survey of some selected recent developments (results and methods) in the theory of Ulam's type stability, such as the Forti method and the methods of fixed points and stability in non-Archimedean spaces. These results and methods have not been treated at all or have been treated only marginally. The very book of Jung [2] covers and offers almost all classic results on the Hyers-UlamRassias stability in an integrated and self-contained fashion. And the authors of [3] discussed Hyers-Ulam stability for functional equations in single variable, including the forms of linear functional equations, nonlinear functional equations, and iterative equations. And they also clarified the relation between Hyers-Ulam stability and other senses of stability which are used for functional equations.

Let $C(X, X)$ be the set of all continuous self-mappings on a topological space $X$. For any $f \in C(X, X)$, let $f^{m}$ denote the $m$ th iteration of $f$; that is, $f^{m}=f \circ f^{m-1}$, $f^{0}=\mathrm{id}, m=1,2, \ldots$ Equations having iterations as their main operation, that is, including iterations of the unknown mapping, are called iterative equations. It is one of the most interesting classes of functional equations [4-7]. As a natural generalization of the problem of iterative roots, iterative equations of the following form:

$$
\begin{array}{r}
\lambda_{1} f(x)+\lambda_{2} f^{2}(x)+\cdots+\lambda_{n} f^{n}(x)=F(x), \\
x \in I=[a, b],
\end{array}
$$

are known as polynomial-like iterative equations. Here $n \geq 2$ is an integer, $\lambda_{i} \in \mathbb{R}(i=1,2, \ldots, n), F: I \rightarrow \mathbb{R}$ is a given mapping, and $f: I \rightarrow I$ is unknown. As mentioned in [8, 9], polynomial-like iterative equations are important not only in the study of functional equations but also in the study of dynamical systems.

In 1986, Zhang [10] constructed an interesting operator called "structural operator" for (1) and used the fixed point theory in Banach space to get the solutions of (1). From then on (1) and other types of equations were discussed extensively by employing this idea (see $[8,9,11-16]$ and references therein). In 2002, by means of a modification of Zhang's method applied in [10], Kulczycki and Tabor [11] investigated the existence of Lipschitzian solutions of the iterative equation

$$
\sum_{i=1}^{\infty} a_{i} f^{i}(x)=F(x), \quad x \in \mathbb{B},
$$

where $\mathbb{B}$ is a compact convex subset of $\mathbb{R}^{N}$ and $F: \mathbb{B} \rightarrow \mathbb{B}$ is a given Lipschitz function. They generalized the results of Zhang [10] to a more general case. 
In 2002, Xu and Zhang [17] discussed the Hyers-Ulam stability for a nonlinear iterative equation which includes the polynomial-like iterative equation. In 2003, Agarwal et al. [3] investigated the Hyers-Ulam stability for a general form of iterative equations which include the polynomial-like iterative equation with variable coefficients. Motivated by the above results, in this paper, we will discuss the Hyers-Ulam stability of (2). As in [11], we give a result on the Hyers-Ulam stability of a functional equation with a more general form firstly. And, by means of this result, the Hyers-Ulam stability of (2) is investigated. In fact, we want to generalize the result of Xu and Zhang [17] to high-dimensional case.

\section{Basic Assumptions, Definitions, and Lemmas}

Let $\mathbb{B}$ be a compact convex subset of $\mathbb{R}^{N}, N \in \mathbb{Z}^{+}$, with nonempty interior. Let

$$
C\left(\mathbb{B}, \mathbb{R}^{N}\right)=\left\{f: \mathbb{B} \longrightarrow \mathbb{R}^{N} \mid f \text { is continuous }\right\} .
$$

In $C\left(\mathbb{B}, \mathbb{R}^{N}\right)$, we use the supremum norm

$$
\|f\|_{\mathbb{B}}=\sup _{x \in \mathbb{B}}\|f(x)\|, \quad \text { for } \forall f \in C\left(\mathbb{B}, \mathbb{R}^{N}\right),
$$

where $\|\cdot\|$ denotes the usual metric of $\mathbb{R}^{N}$. Obviously, $C\left(\mathbb{B}, \mathbb{R}^{N}\right)$ is a complete metric space.

Definition 1 (see [11]). Let $\mathbb{B}$ be a convex compact subset of $\mathbb{R}^{N}$ with nonempty interior. For $m \in[0,1]$, and $M \in[1, \infty]$, one defines

$$
\begin{aligned}
\operatorname{Lip}_{\mathbb{B}}( & m, M) \\
:= & \left\{f: \mathbb{B} \longrightarrow \mathbb{B} \mid f \text { is continuous, }\left.f\right|_{\partial \mathbb{B}}=\left.\mathrm{id}\right|_{\partial \mathbb{B}},\right. \\
& \forall x, y \in \mathbb{B}, \\
& m\|x-y\| \leq\|f(x)-f(y)\| \leq M\|x-y\|\},
\end{aligned}
$$

where $\partial \mathbb{B}$ denotes the boundary of $\mathbb{B}$.

For a given function $f \in C\left(\mathbb{B}, \mathbb{R}^{N}\right)$, we define its Lipschitz constant by

$$
\begin{aligned}
& \operatorname{lip}(f) \\
& \quad:=\inf \{M \geq 0 \mid\|f(x)-f(y)\| \leq M\|x-y\| \forall x, y \in \mathbb{B}\} .
\end{aligned}
$$

Lemma 2 (see [11]). Let $k \in[0,1], K \in[1, \infty]$, and $f \in$ $\operatorname{Lip}_{\mathbb{B}}(k, K)$ be arbitrary. One assumes additionally that $f$ is invertible. Then

$$
f^{-1} \in \operatorname{Lip}_{\mathbb{B}}\left(\frac{1}{K}, \frac{1}{k}\right) .
$$

Lemma 3 (see [11]). For every $m \in(0,1]$, the mapping

$$
\mathscr{I}: \operatorname{Lip}_{\mathbb{B}}(m, \infty) \ni f \longrightarrow f^{-1} \in \operatorname{Lip}_{\mathbb{B}}\left(0, \frac{1}{m}\right)
$$

is well defined with Lipschitz constant $1 / \mathrm{m}$.
Lemma 4 (see [11]). For $K, M<\infty, F \in \operatorname{Lip}_{\mathbb{B}}(0, K)$, the mapping defined by

$$
\mathcal{S}_{F}: \operatorname{Lip}_{\mathbb{B}}(0, M) \ni f \longrightarrow f \circ F \in \operatorname{Lip}_{\mathbb{B}}(0, K M)
$$

is Lipschitz with constant 1.

Lemma 5. Let $m \in[0,1]$, and $M \in[1, \infty]$ be given. For any $f \in \operatorname{Lip}_{\mathbb{B}}(m, M), f: \mathbb{B} \rightarrow \mathbb{B}$ is surjective.

Proof. Note that $\mathbb{B}$ is homeomorphic to $D^{N}$, where $D^{N}=$ $\left\{x \in \mathbb{R}^{N}:\|x\| \leq 1\right\}$ is the unit ball of $\mathbb{R}^{N}$. Without any loss of generality, let $\mathbb{B}=D^{N}$. Since $\left.f\right|_{\partial \mathbb{B}}=\left.\operatorname{id}\right|_{\partial \mathbb{B}}$, set $\left.f\right|_{\partial \mathbb{B}}=f_{0}$; we have $\operatorname{deg}\left(f_{0}\right)=1$, where $\operatorname{deg}\left(f_{0}\right)$ denotes the degree of $f_{0}$. Suppose that $f$ is not surjective. Let $x_{0} \in \mathbb{B} \backslash f(\mathbb{B})$. Since $\left.f\right|_{\partial \mathbb{B}}=\left.\mathrm{id}\right|_{\partial \mathbb{B}}$, we know that $x_{0} \notin \partial \mathbb{B}$; then there exists a retraction mapping $r: \mathbb{B} \backslash\left\{x_{0}\right\} \rightarrow \partial \mathbb{B}$. Thus $r \circ f: \mathbb{B} \rightarrow \partial \mathbb{B}$ is a continuous mapping and $f_{0}=\left.(r \circ f)\right|_{\partial \mathbb{B}}$. This means that $\operatorname{deg}\left(f_{0}\right)=0$, a contradiction. So $f$ is surjective.

Lemma 6. If $f, g$ are homeomorphisms of $\mathbb{B}$ with Lipschitz constant $M$, then

$$
\|f-g\|_{\mathbb{B}} \leq M\left\|f^{-1}-g^{-1}\right\|_{\mathbb{B}} .
$$

Proof. Note that

$$
\begin{aligned}
0 & =\left\|f^{-1} \circ f-g^{-1} \circ g\right\|_{\mathbb{B}} \\
& =\left\|f^{-1} \circ f-g^{-1} \circ f+g^{-1} \circ f-g^{-1} \circ g\right\|_{\mathbb{B}} .
\end{aligned}
$$

So we have

$$
f^{-1} \circ f-g^{-1} \circ f=-g^{-1} \circ f+g^{-1} \circ g \text {. }
$$

Furthermore

$$
\begin{aligned}
\left\|f^{-1}-g^{-1}\right\|_{\mathbb{B}} & =\left\|f^{-1} \circ f-g^{-1} \circ f\right\|_{\mathbb{B}} \\
& =\left\|g^{-1} \circ f-g^{-1} \circ g\right\|_{\mathbb{B}} \\
& \geq \frac{1}{M}\|f-g\|_{\mathbb{B}} .
\end{aligned}
$$

This means that $\|f-g\|_{\mathbb{B}} \leq M\left\|f^{-1}-g^{-1}\right\|_{\mathbb{B}}$.

Lemma 7. Suppose that $f, g: \mathbb{B} \rightarrow \mathbb{B}$ are continuous mappings and $\operatorname{lip}(f)=M$, where $M$ is a positive constant. Then

$$
\left\|f^{k}-g^{k}\right\|_{\mathbb{B}} \leq \sum_{j=0}^{k-1} M^{j}\|f-g\|_{\mathbb{B}}, \quad \forall k=1,2, \ldots
$$


Proof. We use induction to prove this lemma. For $k=1$, $\|f-g\|_{\mathbb{B}} \leq\|f-g\|_{\mathbb{B}}$. Suppose that this is true, for $k$; then

$$
\begin{aligned}
\left\|f^{k+1}-g^{k+1}\right\|_{\mathbb{B}} & \leq\left\|f \circ f^{k}-f \circ g^{k}\right\|_{\mathbb{B}}+\left\|f \circ g^{k}-g \circ g^{k}\right\|_{\mathbb{B}} \\
& \leq M\left\|f^{k}-g^{k}\right\|_{\mathbb{B}}+\|f-g\|_{\mathbb{B}} \\
& \leq M \sum_{j=0}^{k-1} M^{j}\|f-g\|_{\mathbb{B}}+\|f-g\|_{\mathbb{B}} \\
& =\sum_{j=0}^{k} M^{j}\|f-g\|_{\mathbb{B}} .
\end{aligned}
$$

Let $A$ be a subset of $\operatorname{Lip}_{\mathbb{B}}(0, \infty)$ and satisfy

(A1): for all $f, g \in A, f \circ g \in A$;

(A2): if $f \in A$ is invertible, then $f^{-1} \in A$;

(A3): $A$ is closed and convex.

And let

$$
\mathfrak{A}=\left\{A \subset \operatorname{Lip}_{\mathbb{B}}(0, \infty) \mid(\mathrm{A} 1),(\mathrm{A} 2), \text { and }(\mathrm{A} 3) \text { hold }\right\} .
$$

It is easy to see that $\operatorname{Lip}_{\mathbb{B}}(0, \infty) \in \mathfrak{A}$.

Let $A \in \mathfrak{A}$ and let $\mathscr{P}: A \rightarrow A$ be a map. For any $F \in A$ we will consider a functional equation of the following form:

$$
\mathscr{P}(f) \circ f=F,
$$

where $f \in A$ is unknown.

Definition 8. If, for every $g \in \operatorname{Lip}_{\mathbb{B}}(0, \infty)$ such that $\|F-\mathscr{P}(g) \circ g\|_{\mathbb{B}} \leq \delta$, there exists a solution $f$ of (17) such that $\|f-g\|_{\mathbb{B}} \leq K \delta$, where $K>0$ is a constant and $K$ does not depend on the choice of $g$, then (17) is said to have HyersUlam stability.

\section{Main Results}

Lemma 9. Let $\mathbb{B}$ be a convex compact subset of $\mathbb{R}^{N}$ with nonempty interior. Let $A \in \mathfrak{A}$ and $F \in A$ with $\operatorname{lip}(F) \leq M_{0}$. Let $\mathscr{P}: A \rightarrow A$ be given. If there exists a decreasing function $\alpha:[1, \infty] \rightarrow[0,1]$ and a constant $M \in[1, \infty]$ such that

(P1): for any $f \in A, \mathscr{P}(f) \in \operatorname{Lip}_{\mathbb{B}}(\alpha(\operatorname{lip}(f)), \infty)$;

(P2): $M \cdot \alpha(M) \geq M_{0}$.

\section{Then the following two facts hold:}

(c1): for any $f \in A \cap \operatorname{Lip}_{\mathbb{B}}(0, M), \mathscr{P}(f)$ is a homeomorphism with $\operatorname{lip}\left((\mathscr{P}(f))^{-1}\right) \leq 1 / \alpha(M)$;

(c2): for any given $f_{0} \in A \cap \operatorname{Lip}_{\mathbb{B}}(0, M)$, the sequence

$$
f_{k}=\mathcal{S}_{F} \circ \mathscr{I} \circ \mathscr{P}\left(f_{k-1}\right), \quad k=1,2, \ldots,
$$

is well defined and each $f_{k} \in A \cap \operatorname{Lip}_{\mathbb{B}}(0, M)$ is surjective.
Proof. (c1): since $\left.F\right|_{\partial \mathbb{B}}=\left.\mathrm{id}\right|_{\partial \mathbb{B}}$, we get that $M_{0} \geq 1$. By inequality (P2) and the range of the function $\alpha$, we know that both $\alpha(M) \in(0,1]$ and $M \geq M_{0}$ hold. Therefore $F \in A \cap \operatorname{Lip}_{\mathbb{B}}(0, M)$, which implies that $A \cap \operatorname{Lip}_{\mathbb{B}}(0, M)$ is nonempty. For any $x_{1} \neq x_{2} \in \mathbb{B}$ and any $f \in A \cap \operatorname{Lip}_{\mathbb{B}}(0, M)$, since $\operatorname{lip}(f) \leq M$ and $\alpha$ is decreasing, we have

$$
\begin{aligned}
& \left\|\mathscr{P}(f)\left(x_{1}\right)-\mathscr{P}(f)\left(x_{2}\right)\right\| \\
& \quad \geq \alpha(\operatorname{lip}(f))\left\|x_{1}-x_{2}\right\| \geq \alpha(M)\left\|x_{1}-x_{2}\right\|>0 .
\end{aligned}
$$

This means that $\mathscr{P}(f)$ is injective. Since $\mathscr{P}(f) \in A$, by Lemma 5 , we obtain that $\mathscr{P}(f)$ is surjective. Thus $\mathscr{P}(f)$ is a homeomorphism. And $\operatorname{lip}\left((\mathscr{P}(f))^{-1}\right) \leq 1 / \alpha(M)$ follows from Lemma 2.

(c2): by Lemma 3, the mapping

$$
\mathscr{I}: \operatorname{Lip}_{\mathbb{B}}(\alpha(M), \infty) \ni g \longrightarrow g^{-1} \in \operatorname{Lip}_{\mathbb{B}}\left(0, \frac{1}{\alpha(M)}\right)
$$

is continuous. By Lemma 4 and assumptions of $F$, the mapping

$$
\mathcal{S}_{F}: \operatorname{Lip}_{\mathbb{B}}\left(0, \frac{1}{\alpha(M)}\right) \ni g \longrightarrow g \circ F \in \operatorname{Lip}_{\mathbb{B}}\left(0, \frac{M_{0}}{\alpha(M)}\right)
$$

is well defined and continuous.

Now, by the assumption $(\mathbf{P 1})$ and the fact $\mathscr{P}: A \rightarrow A$, it is easy to see that, for every $g \in \operatorname{Lip}_{\mathbb{B}}(0, M) \cap A$,

$$
\mathscr{P}(g) \in \operatorname{Lip}_{\mathbb{B}}(\alpha(M), \infty) \cap A .
$$

Since (A2) holds and by Lemma 2, we know that all elements of $\operatorname{Lip}_{\mathbb{B}}(\alpha(M), \infty)$ are invertible. Moreover we can get that

$$
\mathscr{I} \circ \mathscr{P}(g) \in \operatorname{Lip}_{\mathbb{B}}\left(0, \frac{1}{\alpha(M)}\right) \cap A,
$$

and by means of (A1) we have

$$
\mathcal{S}_{F} \circ \mathscr{I} \circ \mathscr{P}(g) \in \operatorname{Lip}_{\mathbb{B}}\left(0, \frac{M_{0}}{\alpha(M)}\right) \cap A .
$$

Finally, by inequality (P2), we obtain that the mapping

$$
\mathcal{S}_{F} \circ \mathscr{I} \circ \mathscr{P}: \operatorname{Lip}_{\mathbb{B}}(0, M) \cap A \longrightarrow \operatorname{Lip}_{\mathbb{B}}(0, M) \cap A
$$

is well defined.

Take any $f_{0} \in A \cap \operatorname{Lip}_{\mathbb{B}}(0, M)$. By means of the mapping (25), it is easy to see that the sequence

$$
f_{k}=\mathcal{S}_{F} \circ \mathscr{I} \circ \mathscr{P}\left(f_{k-1}\right), \quad k=1,2, \ldots,
$$

is well defined and each $f_{k} \in A \cap \operatorname{Lip}_{\mathbb{B}}(0, M)$. By virtue of Lemma 5 , it is easy to see that all $f_{k}$ are surjective.

Theorem 10. Let $\mathbb{B}$ be a convex compact subset of $\mathbb{R}^{N}$ with nonempty interior. Let $A \in \mathfrak{A}$ and $F \in A$ with $\operatorname{lip}(F) \leq M_{0}$. Let $\mathscr{P}: A \rightarrow A$ be given and let there exist a decreasing function $\alpha:[1, \infty] \rightarrow[0,1]$ and a constant $M \in[1, \infty]$ such that 
(P1) and (P2) of Lemma 9 hold. Moreover, if there is a constant $0<\eta<\alpha(M)$ such that

$$
\begin{array}{r}
\left\|\mathscr{P}\left(g_{1}\right)-\mathscr{P}\left(g_{2}\right)\right\|_{\mathbb{B}} \leq \eta\left\|g_{1}-g_{2}\right\|_{\mathbb{B}}, \\
\forall g_{1}, g_{2} \in A \cap \operatorname{Lip}_{\mathbb{B}}(0, M),
\end{array}
$$

then, for any $g \in A \cap \operatorname{Lip}_{\mathbb{B}}(0, M)$ with

$$
\|F-\mathscr{P}(g) \circ g\|_{\mathbb{B}} \leq \delta,
$$

where $\delta>0$ is a constant, there exists a unique continuous solution $f \in A \cap \operatorname{Lip}_{\mathbb{B}}(0, M)$ of (17) such that

$$
\|f-g\|_{\mathbb{B}} \leq K \delta
$$

where

$$
K=\frac{1}{\alpha(M)-\eta} .
$$

Proof. Since all the assumptions of Lemma 9 hold, so we can construct a sequence $\left\{f_{k}\right\}$ of functions as follows. We take

$$
f_{0}=g
$$

first and then by means of equality (18) repeatedly, define

$$
f_{k}=\mathcal{S}_{F} \circ \mathscr{I} \circ \mathscr{P}\left(f_{k}\right), \quad k=1,2, \ldots
$$

By the conclusions (c1) and (c2) of Lemma 9, both $\mathscr{P}\left(f_{k-1}\right)$ and $f_{k}$ are well defined for all $k>1$. And each $\mathscr{P}\left(f_{k}\right)$ is a homeomorphism of $\mathbb{B}$ onto itself with $\operatorname{lip}\left(\left(\mathscr{P}\left(f_{k}\right)\right)^{-1}\right) \leq$ $1 / \alpha(M)$.

Now we claim that both

$$
\begin{gathered}
\left\|f_{k}-f_{k-1}\right\|_{\mathbb{B}} \leq \frac{1}{\alpha(M)}\left(\frac{\eta}{\alpha(M)}\right)^{k-1} \delta, \\
\left\|F-\mathscr{P}\left(f_{k}\right) \circ f_{k}\right\|_{\mathbb{B}} \leq\left(\frac{\eta}{\alpha(M)}\right)^{k} \delta
\end{gathered}
$$

hold for $k=1,2, \ldots$. We prove these two inequalities inductively.

For $k=1$, by virtue of the definition of $f_{1}$, we have

$$
\begin{aligned}
\| f_{1} & -f_{0} \|_{\mathbb{B}} \\
& =\left\|\mathcal{S}_{F} \circ \mathscr{I} \circ \mathscr{P}\left(f_{0}\right)-f_{0}\right\|_{\mathbb{B}} \\
& =\left\|\left(\mathscr{P}\left(f_{0}\right)\right)^{-1} \circ F-\left(\mathscr{P}\left(f_{0}\right)\right)^{-1} \circ\left(\mathscr{P}\left(f_{0}\right)\right) \circ f_{0}\right\|_{\mathbb{B}} \\
& \leq \frac{1}{\alpha(M)}\left\|F-\left(\mathscr{P}\left(f_{0}\right)\right) \circ f_{0}\right\|_{\mathbb{B}}=\frac{1}{\alpha(M)} \delta .
\end{aligned}
$$

And noting that $F=\mathscr{P}\left(f_{k-1}\right) \circ f_{k}$ and $f_{k}: \mathbb{B} \rightarrow \mathbb{B}$ is surjective for $k=1,2, \ldots$, we have

$$
\begin{aligned}
\left\|F-\mathscr{P}\left(f_{1}\right) \circ f_{1}\right\|_{\mathbb{B}} & =\left\|\mathscr{P}\left(f_{0}\right) \circ f_{1}-\mathscr{P}\left(f_{1}\right) \circ f_{1}\right\|_{\mathbb{B}} \\
& =\left\|\mathscr{P}\left(f_{0}\right)-\mathscr{P}\left(f_{1}\right)\right\|_{\mathbb{B}} \\
& \leq \eta\left\|f_{1}-f_{0}\right\|_{\mathbb{B}}=\frac{\eta}{\alpha(M)} \delta .
\end{aligned}
$$

Assume that they are true for integer $k$. Then, by the same arguments, we can get that

$$
\begin{aligned}
\left\|f_{k+1}-f_{k}\right\|_{\mathbb{B}} & \\
= & \left\|\left(\mathscr{P}\left(f_{k}\right)\right)^{-1} \circ F-\left(\mathscr{P}\left(f_{k}\right)\right)^{-1} \circ\left(\mathscr{P}\left(f_{k}\right)\right) \circ f_{k}\right\|_{\mathbb{B}} \\
& \leq \frac{1}{\alpha(M)}\left\|F-\left(\mathscr{P}\left(f_{k}\right)\right) \circ f_{k}\right\|_{\mathbb{B}} \leq \frac{1}{\alpha(M)}\left(\frac{\eta}{\alpha(M)}\right)^{k} \delta, \\
\| F & -\mathscr{P}\left(f_{k+1}\right) \circ f_{k+1} \|_{\mathbb{B}} \\
& =\left\|\mathscr{P}\left(f_{k}\right) \circ f_{k+1}-\mathscr{P}\left(f_{k+1}\right) \circ f_{k+1}\right\|_{\mathbb{B}} \\
& =\left\|\mathscr{P}\left(f_{k}\right)-\mathscr{P}\left(f_{k+1}\right)\right\|_{\mathbb{B}} \leq \eta\left\|f_{k+1}-f_{k}\right\|_{\mathbb{B}} \\
& =\left(\frac{\eta}{\alpha(M)}\right)^{k+1} \delta .
\end{aligned}
$$

Thus (33) is proved by induction.

For any positive integers $k$ and $s$ with $k>s$, we have

$$
\begin{aligned}
\| f_{k}- & f_{s} \|_{\mathbb{B}} \\
\leq & \left\|f_{k}-f_{k-1}\right\|_{\mathbb{B}}+\left\|f_{k-1}-f_{k-2}\right\|_{\mathbb{B}} \\
& +\cdots+\left\|f_{s+1}-f_{s}\right\|_{\mathbb{B}} \\
\leq & \frac{1}{\alpha(M)}\left(\frac{\eta}{\alpha(M)}\right)^{k-1} \delta+\frac{1}{\alpha(M)}\left(\frac{\eta}{\alpha(M)}\right)^{k-2} \delta \\
& +\cdots+\frac{1}{\alpha(M)}\left(\frac{\eta}{\alpha(M)}\right)^{s} \delta \\
= & \frac{\delta}{\alpha(M)} \cdot \frac{(\eta / \alpha(M))^{s}-(\eta / \alpha(M))^{k}}{1-\eta / \alpha(M)} .
\end{aligned}
$$

Since $\alpha(M)>\eta>0$, it follows from inequality (37) that

$$
\left\|f_{k}-f_{s}\right\|_{\mathbb{B}} \longrightarrow 0, \quad \text { as } k>s \longrightarrow \infty .
$$

As a Cauchy sequence, $\left\{f_{k}\right\}_{k=0}^{\infty}$ converges uniformly in the Banach space $C\left(\mathbb{B}, \mathbb{R}^{N}\right)$. Let

$$
\lim _{k \rightarrow \infty} f_{k}=f
$$

Clearly, $f \in A \cap \operatorname{Lip}_{\mathbb{B}}(0, M)$. From

$$
\begin{aligned}
\|F-\mathscr{P}(f) \circ f\|_{\mathbb{B}} & =\lim _{k \rightarrow \infty}\left\|F-\mathscr{P}\left(f_{k}\right) \circ f_{k}\right\|_{\mathbb{B}} \\
& =\lim _{k \rightarrow \infty}\left(\frac{\eta}{\alpha(M)}\right)^{k} \delta=0,
\end{aligned}
$$


we know that $f$ is a solution of (17). Furthermore,

$$
\begin{aligned}
\| f & -g \|_{\mathbb{B}} \\
& =\lim _{k \rightarrow \infty}\left\|f_{k}-f_{0}\right\|_{\mathbb{B}} \\
& \leq \lim _{k \rightarrow \infty}\left\{\left\|f_{k}-f_{k-1}\right\|_{\mathbb{B}}+\cdots+\left\|f_{1}-f_{0}\right\|_{\mathbb{B}}\right\} \\
& \leq \lim _{k \rightarrow \infty}\left\{\frac{1}{\alpha(M)}\left(\frac{\eta}{\alpha(M)}\right)^{k-1} \delta+\frac{1}{\alpha(M)}\left(\frac{\eta}{\alpha(M)}\right)^{k-2} \delta\right. \\
& \left.+\cdots+\frac{1}{\alpha(M)} \delta\right\} \\
& =\frac{1}{\alpha(M)-\eta} \delta .
\end{aligned}
$$

This proves (29).

Concerning uniqueness, we assume that there is another continuous solution $\phi \in A \cap \operatorname{Lip}_{\mathbb{B}}(0, M)$ for (17), such that

$$
\|\phi-g\|_{\mathbb{B}} \leq \varepsilon
$$

where $\varepsilon>0$ only depends on $\delta$. By Lemma 5 , we know that $F: \mathbb{B} \rightarrow \mathbb{B}$ is also surjective. By means of Lemma 6 and the assumption that $\mathscr{P}$ is Lipschitzian, it follows that

$$
\begin{aligned}
\|f-\phi\|_{\mathbb{B}} & =\left\|(\mathscr{P}(f))^{-1} \circ F-(\mathscr{P}(\phi))^{-1} \circ F\right\|_{\mathbb{B}} \\
& =\left\|(\mathscr{P}(f))^{-1}-(\mathscr{P}(\phi))^{-1}\right\|_{\mathbb{B}} \\
& \leq \frac{1}{\alpha(M)}\|\mathscr{P}(f)-\mathscr{P}(\phi)\|_{\mathbb{B}} \\
& \leq \frac{\eta}{\alpha(M)}\|f-\phi\|_{\mathbb{B}} .
\end{aligned}
$$

This implies that

$$
\left(1-\frac{\eta}{\alpha(M)}\right)\|f-\phi\|_{\mathbb{B}} \leq 0 .
$$

However, $\alpha(M)>\eta>0$; this implies that $\|f-\phi\|_{\mathbb{B}}=0$; that is, $f=\phi$.

\section{Iterative Equations in $\mathbb{R}^{N}$}

Theorem 11. Let $\mathbb{B}$ be a convex compact subset of $\mathbb{R}^{N}$ with nonempty interior. Let $F \in \operatorname{Lip}_{\mathbb{B}}(0, \infty)$ be arbitrary with $\operatorname{lip}(F) \leq M_{0}$. Assume that $a_{k} \in[0,1], k=0,1,2, \ldots$, and there exists a constant $p \in[1, \infty]$ such that

(a1): $\sum_{k=0}^{\infty} a_{k}=1$;

(a2): $a_{0} p>a_{0} p-\sum_{k=1}^{\infty} a_{k} p^{k+1} \geq M_{0}$;

(a3): $\sum_{k=1}^{\infty} a_{k} \sum_{j=0}^{k-1} p^{j}+\sum_{k=1}^{\infty} a_{k} p^{k}<a_{0}$

hold. Then the equation

$$
\sum_{k=0}^{\infty} a_{k} f^{k+1}(x)=F(x), \quad x \in \mathbb{B},
$$

has Hyers-Ulam stability.
Proof. Let $A=\operatorname{Lip}_{\mathbb{B}}(0, \infty)$. For $\forall f \in A$, define

$$
\mathscr{P}(f)=\sum_{k=0}^{\infty} a_{k} f^{k} .
$$

We claim that, for $\forall f \in A, \mathscr{P}(f) \in A$. For $\forall x \in \mathbb{B}$, by the compactness and convexity of $\mathbb{B}$ and the assumption (al), we have

$$
\sum_{k=0}^{\infty} a_{k} f^{k}(x) \in \overline{\operatorname{co}\left\{x, f(x), f^{2}(x), \ldots\right\}} \subset \mathbb{B},
$$

where $\operatorname{co}\left\{x, f(x), f^{2}(x), \ldots\right\}$ is the convex hull of the set $\left\{x, f(x), f^{2}(x), \ldots\right\}$. Moreover, by means of the assumption (al) and the definition of the mapping $\mathscr{P}$ and the set $A$, it is easy to see that $\left.\mathscr{P}(f)\right|_{\partial \mathbb{B}}=\left.\mathrm{id}\right|_{\partial \mathbb{B}}$. Now we prove the continuity of $\mathscr{P}(f)$. By the compactness of $\mathbb{B}$, we first denote

$$
L=\max _{x \in \mathbb{B}}\{\|x\|\}
$$

By the assumption (al), for any $\epsilon>0$, there exists a positive integer $l_{0}$ such that

$$
\sum_{k=l_{0}+1}^{\infty} a_{k} \leq \frac{\epsilon}{4 L}
$$

Note that each $f^{k}, k=0,1,2, \ldots, l_{0}$, is continuous. Then, for any $x \in \mathbb{B}$, there is a $\delta>0$ such that when $\|y-x\|<\delta$ the fact

$$
\left\|f^{k}(y)-f^{k}(x)\right\| \leq \frac{\epsilon}{2\left(l_{0}+1\right)}, \quad k=0,1,2, \ldots, l_{0},
$$

holds. Thus we can get that

$$
\begin{aligned}
\| & \mathscr{P} f(y)-\mathscr{P} f(x) \| \\
& \leq \sum_{k=0}^{l_{0}} a_{k}\left\|f^{k}(y)-f^{k}(x)\right\|+\sum_{k=l_{0}+1}^{\infty} a_{k}\left\|f^{k}(y)-f^{k}(x)\right\| \\
& \leq \sum_{k=0}^{l_{0}} \frac{\epsilon}{2\left(l_{0}+1\right)}+2 L \sum_{k=l_{0}+1}^{\infty} a_{k}=\epsilon .
\end{aligned}
$$

This means that the fact $\mathscr{P}: A \rightarrow A$ holds.

By virtue of the mapping $\mathscr{P},(45)$ can be rewritten as

$$
\mathscr{P}(f) \circ f=F .
$$

Since $\left.F\right|_{\partial \mathbb{B}}=\left.\mathrm{id}\right|_{\partial \mathbb{B}}$, this implies that $M_{0} \geq 1$. And by the assumption (a2), we see that $a_{0}>0$. For $x \in[1, \infty)$, define

$$
\alpha(x)=\max \left\{a_{0}-\sum_{k=1}^{\infty} a_{k} x^{k}, 0\right\} .
$$

Since $\alpha(x) \leq a_{0} \leq 1$ we obtain that $\alpha:[1, \infty] \rightarrow[0,1]$ and it is easy to see that $\alpha$ is decreasing. 
For any $x \in[1, \infty)$ and any $g \in \operatorname{Lip}_{\mathbb{B}}(0, \infty)$ with $\operatorname{lip}(g)=$ $x$, we claim that $\mathscr{P}(g) \in \operatorname{Lip}_{\mathbb{B}}(\alpha(x), \infty)$. It is enough to show that, for $\forall b, c \in \mathbb{B}$,

$$
\alpha(x)\|b-c\| \leq\|\mathscr{P}(g)(b)-\mathscr{P}(g)(c)\| .
$$

But

$$
\begin{aligned}
& \|\mathscr{P}(g)(b)-\mathscr{P}(g)(c)\| \\
& =\left\|\sum_{k=0}^{\infty} a_{k}\left(g^{k}(b)-g^{k}(c)\right)\right\| \\
& \geq a_{0}\|b-c\|-\sum_{k=1}^{\infty} a_{k}\left\|g^{k}(b)-g^{k}(c)\right\| \\
& \geq a_{0}\|b-c\|-\sum_{k=1}^{\infty} a_{k} x^{k}\|b-c\| \\
& =\left(a_{0}-\sum_{k=1}^{\infty} a_{k} x^{k}\right)\|b-c\|=\alpha(x)\|b-c\| .
\end{aligned}
$$

It follows from the assumption (a2) that

$$
p \cdot \alpha(p)=a_{0} p-\sum_{k=1}^{\infty} a_{k} p^{k+1} \geq M_{0}
$$

Thus (P1) and (P2) of Lemma 9 also hold. Moreover, we can get that

$$
\begin{aligned}
\|\mathscr{P}(f)-\mathscr{P}(g)\|_{\mathbb{B}} & =\left\|\sum_{k=0}^{\infty} a_{k}\left(f^{k}-g^{k}\right)\right\|_{\mathbb{B}} \\
& \leq \sum_{k=0}^{\infty} a_{k}\left\|f^{k}-g^{k}\right\|_{\mathbb{B}} \\
& \leq \sum_{k=1}^{\infty} a_{k} \sum_{j=0}^{k-1} p^{j}\|f-g\|_{\mathbb{B}}
\end{aligned}
$$

hold. Let $\eta=\sum_{k=1}^{\infty} a_{k} \sum_{j=0}^{k-1} p^{j}$. By the assumption (a2), we know that there must be a positive integer $k_{0}$ such that $a_{k_{0}} \neq 0$. So we know that $\eta>0$. Furthermore, by the assumption (a3), we have $0<\eta<\alpha(p)$. Then, by Theorem 10, we get the conclusion.

Example 12. For $F(x)=\begin{array}{r}\left(x_{1}, x_{2}, x_{3}, \ldots, x_{N}\right) \\ +\operatorname{dist}\left(x, S^{N-1}\right)(1,0,0, \ldots, 0) \\ \left.\sqrt{x_{1}^{2}+x_{2}^{2}+\cdots+x_{N}^{2}}, x_{2}, \ldots, x_{N}\right),\end{array} \quad \begin{gathered}\left(1+x_{1}-\right. \\ \text { where }\end{gathered} \quad x \quad$ $\left(x_{1}, x_{2}, \ldots, x_{N}\right) \in D^{N}$ and $\operatorname{dist}\left(x, S^{N-1}\right)$ denotes the distance of the point $x$ from $S^{N-1}$, obviously, $\left.F\right|_{S^{N-1}}=\left.\mathrm{id}\right|_{S^{N-1}}$. By simple calculation, we get that, for any $x, y \in D^{N}$, the following two inequalities:

$$
\begin{gathered}
\|F(x)-F(y)\| \leq 2\|x-y\|, \\
\|F(x)\| \leq\|x\|+\operatorname{dist}\left(x, S^{N-1}\right)=1
\end{gathered}
$$

hold. This implies that $F \in \operatorname{Lip}_{D^{N}}(0,2)$. Then we consider the equation

$$
\begin{gathered}
\frac{254}{255} f(x)+\sum_{i=1}^{\infty}\left(\frac{1}{256}\right)^{i} f^{i+1}(x)=F(x), \quad x \in D^{N}, \\
F: D^{N} \longrightarrow D^{N},\left.\quad F\right|_{S^{N-1}}=\left.\mathrm{id}\right|_{S^{N-1}} .
\end{gathered}
$$

Denote $a_{0}=254 / 255, a_{k}=(1 / 256)^{i}, k=1,2, \ldots$. Define $\alpha(x)=\max \left\{(254 / 255)-\sum_{i=1}^{\infty}(1 / 256)^{i} x^{i}, 0\right\}$. Let $M_{0}=2$ and $p=3$. Then we have

$$
\begin{gathered}
\sum_{k=0}^{\infty} a_{k}=1, \quad \frac{254}{255} \times 3>3 \alpha(3)=\frac{190491}{64515}>2 \geq \operatorname{lip}(F), \\
\frac{254}{255}>\sum_{k=1}^{\infty}\left(\frac{1}{256}\right)^{k} \sum_{j=0}^{k-1} 3^{j}+\sum_{i=1}^{\infty}\left(\frac{3}{256}\right)^{i}=\frac{1021}{64515}
\end{gathered}
$$

By Theorem 11, (59) has Hyers-Ulam stability.

\section{Conflict of Interests}

The authors declare that there is no conflict of interests regarding the publication of this paper.

\section{Acknowledgment}

The research of the authors was supported by National Natural Science Foundation of China (Grant nos. 11101105 and 11001064).

\section{References}

[1] N. Brillouët-Belluot, J. Brzdęk, and K. Ciepliński, "On some recent developments in Ulam's type stability," Abstract and Applied Analysis, Article ID 716936, 41 pages, 2012.

[2] S.-M. Jung, Hyers-Ulam-Rassias Stability of Functional Equations in Nonlinear Analysis, vol. 48 of Springer Optimization and Its Applications, Springer, New York, NY, USA, 2011.

[3] R. P. Agarwal, B. Xu, and W. Zhang, "Stability of functional equations in single variable," Journal of Mathematical Analysis and Applications, vol. 288, no. 2, pp. 852-869, 2003.

[4] M. Kuczma, B. Choczewski, and R. Ger, Iterative Functional Equations, Encyclopedia of Mathematics and Its Applications, Cambridge University Press, 1990.

[5] K. Baron and W. Jarczyk, "Recent results on functional equations in a single variable, perspectives and open problems," Aequationes Mathematicae, vol. 61, no. 1-2, pp. 1-48, 2001.

[6] C. Nabeya, "On the functional equation $f(p+q x+r f(x))=$ $a+b x+c f(x)$," Aequationes Mathematicae, vol. 11, no. 2-3, pp. 199-211, 1974.

[7] J. Z. Zhang, L. Yang, and W. N. Zhang, Iterative Equations and Embedding Flow, Shanghai Scientific and Technological Education, 1998.

[8] W. N. Zhang, K. Nikodem, and B. Xu, "Convex solutions of polynomial-like iterative equations," Journal of Mathematical Analysis and Applications, vol. 315, no. 1, pp. 29-40, 2006. 
[9] B. Xu and W. Zhang, "Decreasing solutions and convex solutions of the polynomial-like iterative equation," Journal of Mathematical Analysis and Applications, vol. 329, no. 1, pp. 483497, 2007.

[10] W. N. Zhang, "On the differentiable solutions of the iterated equation $\sum_{i=1}^{n} \lambda_{i} f^{i}(x)=F(x)$," Chinese Science Bulletin, vol. 32, no. 21, pp. 1444-1451, 1987.

[11] M. Kulczycki and J. Tabor, "Iterative functional equations in the class of Lipschitz functions," Aequationes Mathematicae, vol. 64, no. 1-2, pp. 24-33, 2002.

[12] V. Murugan and P. V. Subrahmanyam, "Existence of solutions for equations involving iterated functional series," Fixed Point Theory and Applications, vol. 2005, no. 2, pp. 219-232, 2005.

[13] V. Murugan and P. V. Subrahmanyam, "Special solutions of a general iterative functional equation," Aequationes Mathematicae, vol. 72, no. 3, pp. 269-287, 2006.

[14] V. Murugan and P. V. Subrahmanyam, "Special solutions of a general iterative functional equation," Aequationes Mathematicae, vol. 72, no. 3, pp. 269-287, 2006.

[15] V. Murugan and P. V. Subrahmanyam, "Special solutions of a general iterative functional equation," Aequationes Mathematicae, vol. 76, no. 3, pp. 317-320, 2008.

[16] D. L. Yang and W. N. Zhang, "Characteristic solutions of polynomial-like iterative equations," Aequationes Mathematicae, vol. 67, no. 1-2, pp. 80-105, 2004.

[17] B. Xu and W. N. Zhang, "Hyers-Ulam stability for a nonlinear iterative equation," Colloquium Mathematicum, vol. 93, no. 1, pp. $1-9,2002$. 


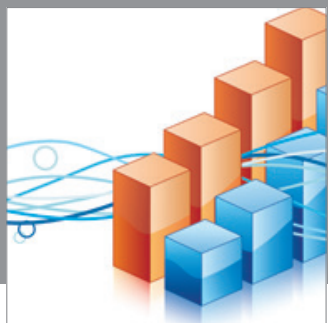

Advances in

Operations Research

mansans

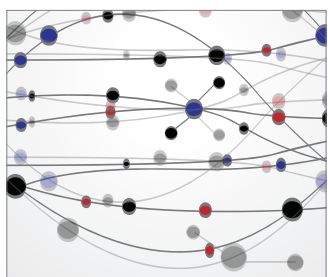

The Scientific World Journal
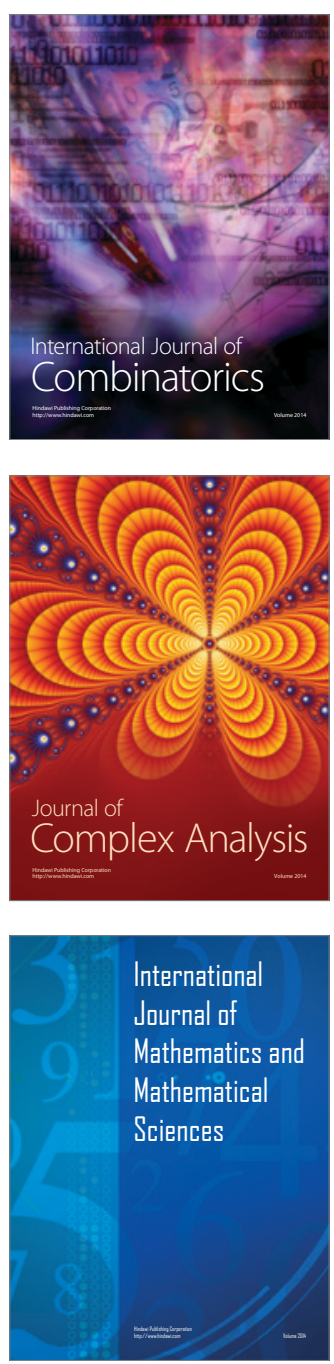
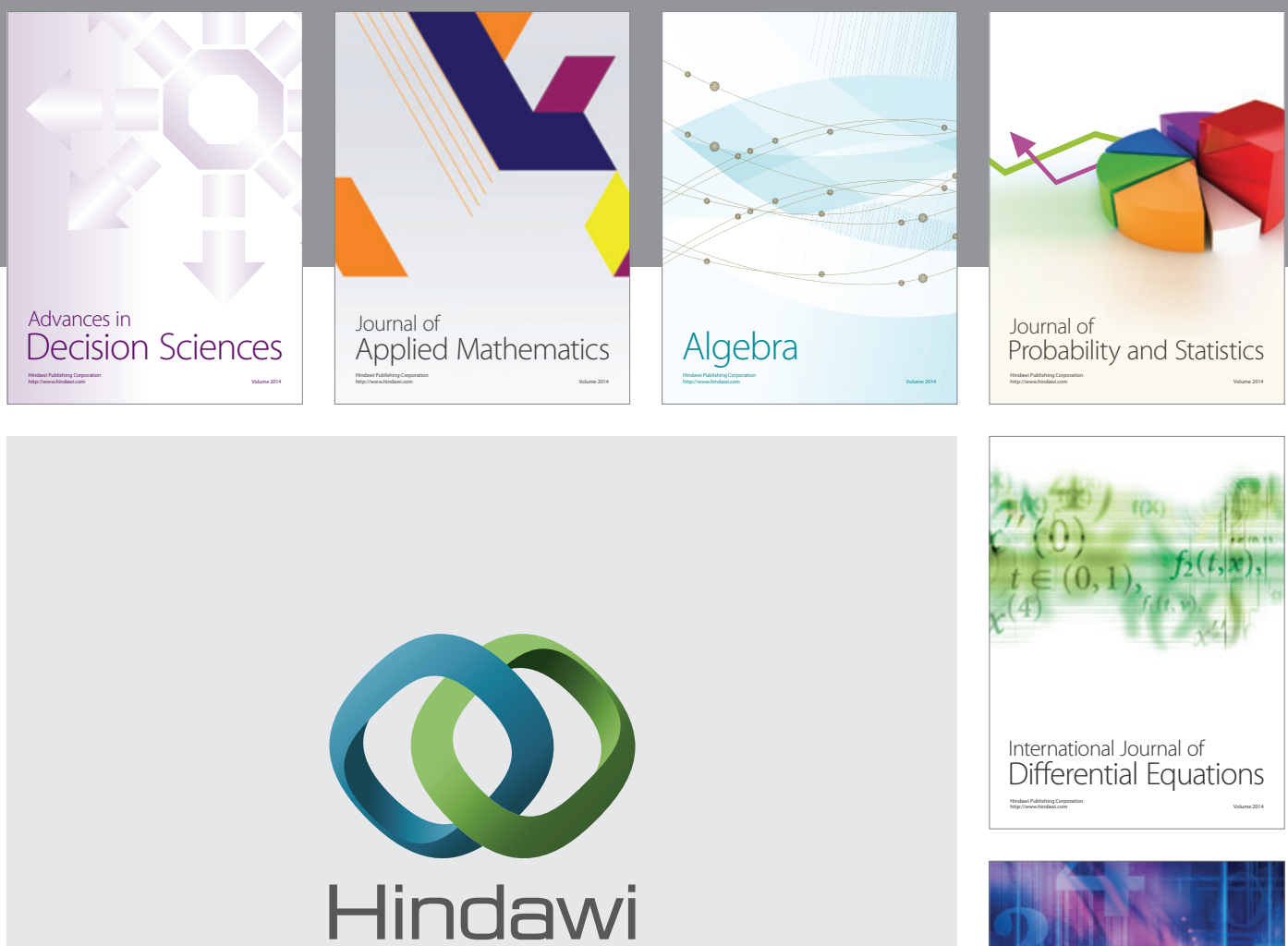

Submit your manuscripts at http://www.hindawi.com
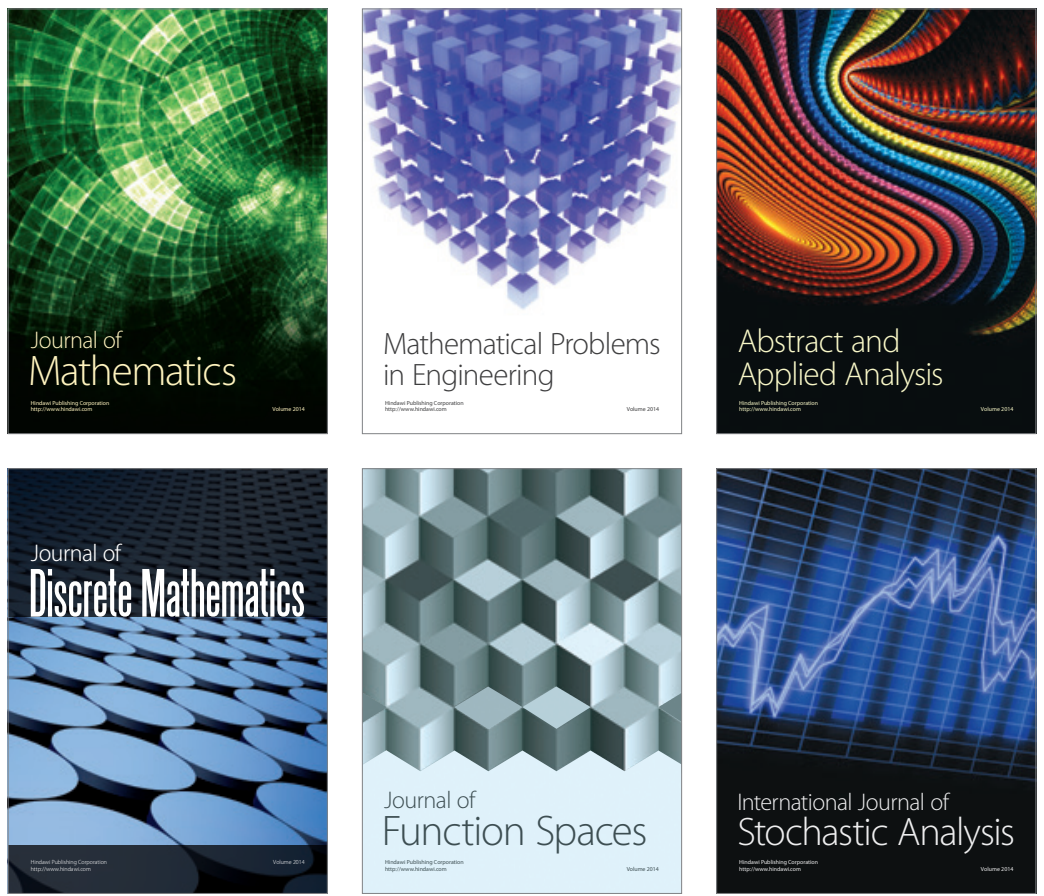

Journal of

Function Spaces

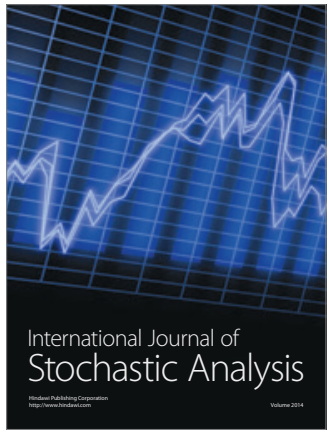

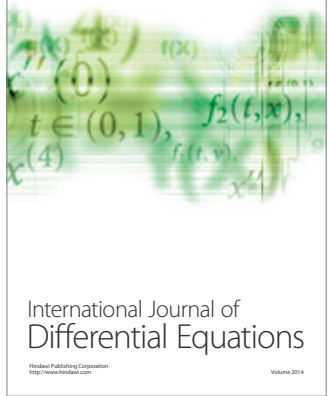
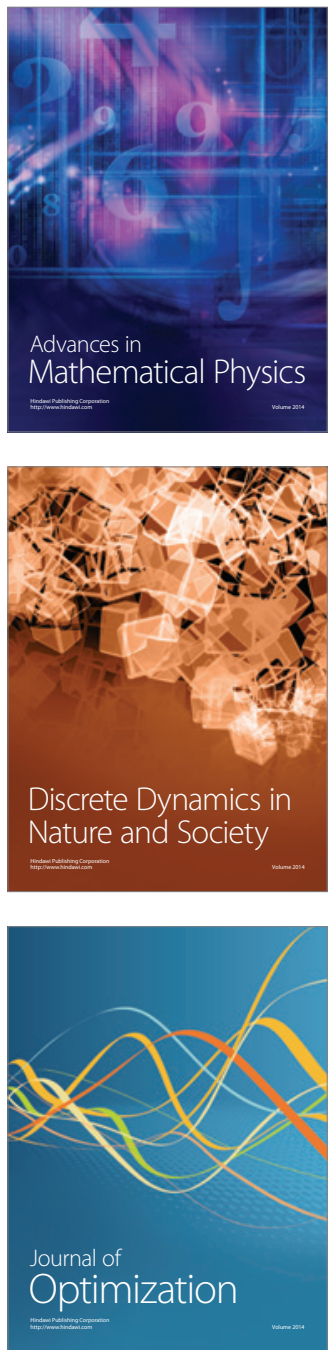